\title{
Ni sang ni sol ?Crise de l'Europe et dialectique de la territorialité
}

Pierre Hassner

\section{(2) OpenEdition \\ 12 Journals}

Édition électronique

URL : http://journals.openedition.org/conflits/259

DOI : $10.4000 /$ conflits.259

ISSN : $1777-5345$

Éditeur :

CCLS - Centre d'études sur les conflits lilberté et sécurité, L'Harmattan

Édition imprimée

Date de publication : 15 mai 1996

ISSN : 1157-996X

Référence électronique

Pierre Hassner, « Ni sang ni sol ?Crise de l'Europe et dialectique de la territorialité », Cultures \& Conflits [En ligne], 21-22 I printemps-été 1996, mis en ligne le 15 mars 2006, consulté le 30 mars 2021. URL : http://journals.openedition.org/conflits/259; DOI : https://doi.org/10.4000/conflits.259

Ce document a été généré automatiquement le 30 mars 2021.

Creative Commons License 


\title{
Ni sang ni sol ?Crise de l'Europe et dialectique de la territorialité
}

\author{
Pierre Hassner
}

1 «Rome, l'unique objet de mon ressentiment !... « Rome n'est plus dans Rome, elle est toute où je suis ».

2 Ces deux exclamations cornéliennes résument bien les réactions actuellement les plus retentissantes, sinon les plus répandues, à l'égard de l'Europe. D'un côté, l'Europe, bouc émissaire de la mondialisation et de la crise d'identité des nations occidentales, de la tyrannie des marchés et des contraintes de la rigueur, du chômage et de l'insécurité, de la concurrence des bas salaires lointains et de la présence troublante des immigrés. De l'autre, l'Europe, centre mythique dont on se réclame et auquel on aspire, dont on se prétend le représentant ou le défenseur au milieu des barbares, et par lequel on se sent rejeté, abandonné, ou marginalisé. Mais s'agit-il de la même Europe ? Et, que met-on sous ce nom? Une entité géographique ou historique, économique ou politique? Que couvre-t-elle, en extension et en compréhension? Ni terroir, terre maternelle ou Heimat, ni cyberspace mondial, ni communauté affective, ni réseau universel, est-elle, comme l'État national, liée à la dimension intermédiaire du territoire et, à ce titre, représente-t-elle un compromis, entre la famille ou le groupe primaire et, la société mondiale impersonnelle et incontrôlable ? Ou perd-elle sur les deux tableaux, comme le " territoire » lui-même, lié à des structures d'administration, d'autorité ou de légitimité impuissantes ou contestées? Que la crise de l'Europe soit, par certains côtés, une version particulière, et particulièrement aiguë, de la crise de la territorialité ou du moins qu'elle soit traversée, ou portée, par les paradoxes et les contradictions de ce que Marie-Françoise Durand a appelé « l'articulation du territoire et des réseaux " ${ }^{1}$, ellemême liée, en dernière analyse, à celle de la société mondiale et des communautés particulières, c'est ce qu'illustrent trois exemples qui inspireront et orienteront notre exposé. Le premier concerne le problème des frontières de l'Union européenne, tel qu'il est posé par l'éternel débat sur son élargissement. Où s'arrête la construction européenne ? En 1954, polémiquant contre la " petite Europe », François Perroux avait lancé la formule de "l'Europe sans rivages ». Mais la véritable «Europe sans rivages », 
n'est-ce pas le monde ? Peut-on vider l'intégration européenne de toute limite, voire de toute référence géographique territoriale? Mais, inversement, comment considérer celles de l'Europe rhénane ou celles de l'Europe occidentale, définie par le rideau de fer, ou de l'Europe de l'Atlantique à l'Oural définie par une géographie étrangère aux réalités politiques et économiques du XXe siècle, comme définitives? Il semble bien que l'Europe de la construction européenne soit condamnée à rester ambiguë par rapport à la géographie, qu'elle ne puisse se penser sans frontières, mais que ces frontières soient condamnées à rester mouvantes et contradictoires. Plus à l'Est, c'est surtout un autre paradoxe qui est le plus sensible : celui des rapports entre la sécurité européenne et le problème des frontières. Le principe proclamé par les accords d'Helsinki est celui de l'intangibilité des frontières et de la libre circulation des hommes et des idées, donc, celui des frontières à la fois fixes et perméables. Or c'est le contraire qui se produit. Du fait de la désintégration des États communistes multinationaux, 14. $200 \mathrm{~km}$ de frontières nouvelles sont créées depuis 1989. Certes, celles-ci retrouvent d'anciennes frontières historiques et, même dans le cas de l'exYougoslavie, l'idée du maintien de la Bosnie-Herzégovine dans ses frontières internationalement reconnues est maintenue, mais elle est contredite par sa division de fait, produite par la conquête. D'autre part, cette même guerre d'ex-Yougoslavie nous montre une réaffirmation exacerbée du territoire : villes interminablement assiégées ou divisées, zones de sécurité, enclaves, corridors, négociations minutieuses sur les enjeux les plus classiques tels que l'accès à la mer, etc. Qui n'a remarqué le paradoxe selon lequel les frontières tendent à s'effacer à l'Ouest, traditionnellement divisé en États nationaux, et à s'élever à l'Est, traditionnellement impérial ? Pierre Manent a remarqué que «l'inconscience territoriale de l'Europe de l'Ouest fait un pendant désastreux à l'hyperesthésie territoriale de l'Europe de l'Est " ${ }^{2}$. Encore faut-il ne pas oublier que l'Est n'est pas étranger aux migrations ni l'Ouest aux fermetures - qu'il s'agisse des divisions linguistiques en Belgique, de la fermeture des pays occidentaux aux immigrants ou des tendances à la ghettoisation ou à la séparation territoriale des communautés à l'intérieur des grandes métropoles. La dialectique mondiale de la globalisation et de la fragmentation semble aboutir à ce que plus les communications deviennent faciles, plus la séparation territoriale sur le modèle de Chypre ou d'Israël semble l'emporter sur la coexistence dans la diversité. Notre époque semble redécouvrir à la fois le nomadisme, l'existence d'unités politiques mobiles ou nonterritoriales, et le pré carré, la volonté forcenée de rester entre soi. Et cela nous amène précisément au troisième exemple qui concerne à la fois l'Est et l'Ouest, le Nord et le Sud : le problème des réfugiés. A l'heure où, par la disparition du rideau de fer, et par la multiplication des guerres, des famines, et des nettoyages ethniques, des millions d'êtres humains en Europe, des dizaines de millions ailleurs, sont amenés à chercher refuge hors de leurs frontières, les pays d'accueil se ferment, poussés par leur crise économique et leur crise d'identité. On a pu dire que les migrations témoignaient de la dévalorisation du territoire. Inversement, chassés de leur territoire et refoulés de ceux auxquels ils aspirent, les "réfugiés sur orbite» témoignent par la négative de l'importance du territoire. La géographie des migrations forcées, depuis le nettoyage ethnique à un bout, jusqu'à l'immigration clandestine et sa répression à l'autre, est à l'origine de réseaux économiques qui rivalisent avec ceux du commerce des armes et de la drogue. L'Europe y joue un rôle de plaque tournante qui suggère, comme V. Andreff pour les sociétés multinationales ${ }^{3}$, qu'on ne peut comprendre la nouvelle réalité du territoire qu'en termes non pas de disparition mais de différenciation. C'est cette 
différenciation que nous allons décrire, dans le cas de l'Europe, en allant d'Ouest en Est et de l'abstrait au concret, avant de revenir au problème plus général que nous venons d'évoquer.

3 L'Union Européenne Nous avons déjà parlé de l'ambiguïté essentielle de la construction européenne quant à ses limites géographiques. Elle s'étend à la nature même de l'entreprise et surtout de l'entité qui en résulte, au point que Jacques Delors a pu parler "d'objet non identifié », et le politiste américain John Ruggie de «la première entité politique à perspectives multiples " ${ }^{4}$. Si, comme il le suggère, on considère que l'attribut le plus fondamental de tout système de gouvernement est « la domination légitime sur une étendue spatiale " et qu'à l'intérieur de cette définition ce qui caractérise la modernité c'est " une forme particulière de territorialité " ${ }^{5}$, alors, il faut considérer que le caractère flou et mobile des rapports entre l'intérieur et l'extérieur, l'intergouvernemental et le supra-national, le politique et le fonctionnel qui caractérise l'Union européenne est en rupture avec cette modernité. D'où le débat central depuis et autour du traité de Maastricht: le caractère hybride de l'entité européenne est-il pathologique et provisoire, doit-il faire place à un retour vers la coopération intergouvernementale pure et simple ou à un progrès vers un État, voire un État-nation européen, ou du moins vers une fédération aux compétences bien définies? Ou correspond-il, au contraire, à un caractère même de la réalité socio-politique, nationale, internationale et transnationale dans laquelle nous entrons, et qui serait caractérisée, par l'interpénétration des unités et des domaines, la multiplicité des allégeances et des compétences, et leur mobilité ou leur fluidité ? Certainement la multiplication des formules paradoxales ou intermédiaires pour décrire les rapports entre organisations et États membres, candidats ou associés indique une prédominance du dégradé et de l'ambiguïté sur les délimitations claires et distinctes qui doit avoir un rapport avec la dévalorisation du territoire par l'urbanisation, avec la multiplication des réseaux, et les difficultés du contrôle exclusif et de la fermeture. Mais, par un mouvement dialectique que nous retrouverons tout au long de cet article, cette fluidité est déstabilisatrice et cette déstabilisation par l'ouverture produit des effets de fermeture et de retour à la rigidité. La relation entre le centre et la périphérie est certes ambiguë et mouvante au niveau de l'Union européenne, au point que l'on est obligé de distinguer entre périphérie intérieure et extérieure, et que la distinction entre centre et périphérie tend à se reproduire à l'intérieur du centre et de la périphérie elle-même par un dégradé qui va depuis le centre du centre jusqu'à la périphérie de la périphérie. Mais le point essentiel est qu'il ne s'agit ni d'une hiérarchie stable et acceptée ni d'une tendance à l'effacement pur et simple des distinctions. On assiste plutôt à un champ de forces où l'attraction et la répulsion s'exercent de façon simultanée et contradictoire. Il y a une tendance au repli du centre par rapport aux périphéries turbulentes et violentes. Mais, dans le cas de la fermeture à l'immigration, cette tendance joue, certes, en faveur de la création d'un fossé ou d'un mur entre l'intérieur et l'extérieur, symbolisé par l'accord de Schengen, mais on constate en même temps un repli des États-membres sur eux-mêmes et la création de nouveaux espaces et de nouvelles différenciations, à l'intérieur et à l'extérieur, allant des zones de sécurité dans les aéroports aux «pays de premier accueil » ou " aux premiers pays sûrs ». D'autre part, la «sécession des nantis» se reproduit à l'intérieur des États (par l'opposition des régions riches et des régions pauvres, des régions ouvertes sur l'extérieur et des régions marginales), voire des villes qui commencent une re-territorialisation par l'opposition des beaux quartiers protégés et des banlieues (ou au contraire des inner 
cities) à risques. Cette opposition elle-même peut, d'ailleurs, se renverser dans le cas des rapports entre le centre (européen ou atlantique) et la périphérie orientale ou méridionale. Le calendrier de l'Union européenne comme de l'OTAN sont dominés par le problème de l'élargissement à l'Europe centrale dont les États sont des candidats plus plausibles, parce que plus proches, économiquement, politiquement ou culturellement et donc plus intégrables que leurs voisins à l'Est et au Sud. Mais les mêmes raisons pour lesquelles l'Occident prospère et libéral souhaiterait tenir à distance une région comme les Balkans (le désordre, la guerre, la famine) l'amènent à s'y intéresser malgré lui. Paradoxalement, après l'accord de Dayton, l'Union européenne et l'OTAN seront beaucoup plus engagées en tant que telles dans les Balkans (en particulier, mais pas exclusivement, par l'effort de reconstruction en ex-Yougoslavie) que dans cette Europe centrale plus familière mais moins en état d'urgence, dont la priorité pourrait encore reculer. Le cas des États baltes, dont la dimension et les affinités nordiques permettraient une intégration rapide sur le plan économique, mais auxquels leurs rapports géopolitiques et historiques avec la Russie interdisent d'envisager leur adhésion à l'OTAN ou leur protection par elle, est une autre illustration paradoxale de la discordance entre les degrés de proximité ou de distance selon les domaines.

États et régions Cette multiplicité et cette fluidité des dimensions entraînent inévitablement une relativisation de l'État territorial national. Mais cette dernière est vécue de manière différente par les différents pays. Certes la liaison des trois termes de territoire, d'État et de nation est constitutive de la modernité. Il n'y a pas aujourd'hui d'États sans territoire, et l'ambiguïté du terme de nation permet à tous les États territoriaux, même multinationaux, de prétendre à un patriotisme ou à un sentiment national, fondé sur la citoyenneté. Mais certains États, comme la France, incarnent davantage la modernité, tandis que d'autres, comme l'Allemagne, tiennent peut-être de leur héritage pré-moderne une plus grande facilité d'adaptation à la crise de la modernité, voire à l'avènement de l'âge post-moderne. En France, si l'idéologie, longtemps dominante, des frontières naturelles est abandonnée, celle des frontières fixes et cohérentes ne l'est pas. Michel Feher a pu parler de l'opposition des " démocrates sans frontières » et des "républicains en un seul pays »; il est clair que ce sont les seconds qui sont le plus conformes à la tradition jacobine française. Ils sont, aujourd'hui, portés par la réaction contre le traité de Maastricht et, plus profondément, contre le marché mondial et l'ouverture des frontières. Sur le plan théorique, Blandine Barret-Kriegel s'est faite l'avocate, au nom de l'État de droit, de la continuité entre la monarchie française et la République, opposée à la continuité entre l'empire et l'intégration européenne, domaine du flou et de l'indéfini, donc dangereuse pour le droit et la démocratie ${ }^{6}$. Inversement, l'historien allemand Reinhart Koselleck soutient que l'Allemagne, n'ayant jamais été vraiment un État national (à part une exception malheureuse de soixante-dix ans), mais ayant toujours connu la multiplicité et l'entrelacement des types d'unités politiques et d'allégeance, des corporations aux Länder en passant par les principautés, se trouve d'autant plus désignée non seulement pour s'adapter à une Europe fédérale mais pour lui servir de modèle ${ }^{7}$.. Et de fait, les régions sub et transnationales sont souvent vues en France comme une menace à la souveraineté et à l'autorité de l'État ou comme l'introduction d'échelons supplémentaires de bureaucratie et de corruption, alors qu'en Allemagne les Länder constituent une réalité incontournable, vivante et puissante, encore que, peut-être, déclinante, et les régions transnationales ou européennes, une occasion de prospérité et aussi, peut-être, d'influence ou d'hégémonie. Les attitudes des deux pays envers 
l'approfondissement et l'élargissement de l'Union européenne correspondent à ce schéma d'une préférence française pour l'État et le statu quo et d'une plus grande confiance allemande en la dynamique des sociétés et des institutions non-étatiques. Une certaine logique aurait voulu que les partisans de l'élargissement soient, comme les Anglais, hostiles à l'approfondissement et que les partisans de celui-ci soient, comme les fédéralistes européens classiques, hostiles à l'élargissement. Mais la France, tout en étant en principe favorable à l'un et à l'autre, est en réalité réticente, ou sur la défensive, dans les deux cas, devant la perte de contrôle gouvernemental et, peut-être, devant l'accroissement de l'influence allemande qu'ils impliquent. Effectivement, les milieux dirigeants allemands (plus que l'opinion publique qui renâcle, dans chaque cas, à payer davantage pour une stabilité moins garantie) parient à la fois sur l'approfondissement dans la direction fédérale et sur l'élargissement à l'Europe centrale. Il n'est pas interdit de penser que l'idée d'une souveraineté européenne sur des régions telles que la Silésie ne soit, consciemment ou inconsciemment, considérée comme une possibilité de réunion effective à l'Allemagne dans le respect des frontières reconnues et « au nom de l'Europe ${ }^{8}$. La France est à la pointe en matière européenne lorsqu'il s'agit de coopération intergouvernementale en matière de défense. L'Allemagne porte son attention avant tout sur les domaines économique et culturel. Raymond Aron citait, pendant la guerre, un penseur pacifiste allemand, W. Foerster, pour qui le malheur de l'Allemagne et de l'Europe tenait à ce que l'unité allemande ait été réalisée sous la direction de la Prusse, c'est-à-dire par le fer et par le feu. Selon lui, la situation géographique de l'Allemagne et son poids économique et démographique rendaient une certaine hégémonie allemande sur l'Europe inévitable ; mais la solution petite-allemande, sous l'égide de la Prusse, tendait à la concevoir sous la forme d'une domination brutale, politico-militaire, qui ne pouvait qu'être rejetée par ses voisins. Une solution grande-allemande inspirée du Saint Empire Romain Germanique qui aurait respecté la dignité, la souveraineté et l'égalité formelle de tous, mais exercé une influence économique et culturelle prédominante, au nom de l'intérêt commun aurait $\mathrm{pu}$, au contraire, être acceptée ${ }^{9}$. Peut-être n'est-ce pas là, la plus mauvaise clé d'interprétation pour la politique de Bonn. N'a-t-on pas pu dire récemment que, « dans le fond, le chancelier Kohl a réussi à obtenir pacifiquement ce que d'autres que lui ont essayé d'extorquer par la conquête armée depuis Bismarck: une zone de paix et de prospérité tout autour de l'Allemagne ${ }^{10}$ ? Encore faut-il bien voir ce que cette notion de « zone autour de l'Allemagne » implique au point de vue de la question du territoire, à savoir d'une part son importance et d'autre part sa relativisation. L'Allemagne peut exercer un rôle prédominant en Europe centrale et dans les Balkans grâce à sa proximité géographique et à sa présence historique; elle tient à l'inclusion de la Pologne, en particulier, dans les organisations occidentales, parce qu'elle ne veut plus être la frontière orientale de l'Occident mais veut être entourée d'États démocratiques et alliés; mais elle ne peut y arriver que dans la mesure où d'une part elle accepte sans équivoque ses frontières politiques actuelles, et renonce à toute expansion territoriale, et d'autre part elle se situe dans un cadre plus large, celui de l'Union européenne.

5 Nations et empires Cette dialectique allemande pourrait être étendue, ne serait-ce que par analogie, aux deux autres États-clés, héritiers des deux autres empires, qui sont tiraillés, par leur géographie et par leur histoire, entre une version nationale et une version impériale de leur identité : la Turquie et la Russie. Si la France et l'Angleterre ont, elles aussi, connu l'opposition entre «l'hexagone » et «la plus grande France » ou entre "Great Britain and Little England», c'est outre-mer, du côté des empires 
coloniaux ou des communautés de langue que se sont portées les visions impériales ou globalisantes, désormais affaiblies de manière décisive. Pour les héritiers des empires continentaux, la question se pose de manière beaucoup plus intime et actuelle, du moins aux yeux de leurs voisins toujours prompts à déceler une résurrection de pangermanisme, de panturquisme et du panslavisme. Dans les cas allemand et turc, la république de Bonn et, bien avant, l'État kémaliste ont cherché à donner une nouvelle base à leur identité en rompant de manière radicale avec la nostalgie du passé impérial. L'effondrement de l'empire russo-communiste combiné avec les déceptions de l'intégration européenne peut ouvrir des solutions de rechange au "patriotisme constitutionnel» et à l'idéologie républicaine. Certains craignent qu'avec la réunification l'Allemagne ne redevienne une nation plus qu'une société, et que de l'identité nationale aux ambitions de puissance et de celles-ci aux visées impériales il n'y ait qu'un pas. En Turquie, la présidence Ozal a semblé ouvrir, par opposition à l'État national, unitaire et centralisé d'Ataturk, une perspective plus souple et plus large inspirée de l'empire ottoman, faisant place à la fois à plus d'autonomie pour les minorités intérieures comme les Kurdes et à plus de solidarité avec (ou d'influence sur et à travers) les populations turques ou musulmanes des Balkans, du Caucase et d'Asie centrale. Mais cette vision s'est heurtée très rapidement aux limites actuelles de la puissance turque et à la conscience des dangers de réaction russe et de méfiance occidentale. Aujourd'hui, la priorité nationale et européenne reste valable, la prudence prévaut entièrement au point de vue militaire et, comme pour l'Allemagne, la quête d'influence régionale se situe presque exclusivement sur le plan économique et culturel. A la différence de l'Allemagne, cependant, les réactions de dépit envers l'Europe et la renaissance de l'islamisme peuvent amener des révisions déchirantes. La situation est encore bien moins claire pour ce qui concerne la Russie. Celle-ci n'a jamais opéré le passage radical de l'Empire à l'État que la défaite militaire ou la volonté d'un leader ont imposé à l'Allemagne et à la Turquie. Comme cela a souvent été remarqué, son identité est impériale avant d'être nationale. Les grands espaces et les frontières mouvantes semblent faire partie de sa perception identitaire. Là où pour l'Allemagne et même la Turquie il faut scruter les reins, les coeurs et l'avenir pour trouver des ambiguïtés inquiétantes et déstabilisatrices, en Russie ce sont les affirmations claires d'une nouvelle identité nationale et étatique, et d'une rupture avec le passé impérial, qu'il faut chercher à la loupe. Ce qu'on trouve, au contraire, c'est une ambiguïté constante entre frontières russes et soviétiques, aire slave et solidarité orthodoxe, "étranger proche» et sphère d'influence, rôle européen et asiatique, etc.. C'est le Maréchal Gratchev déclarant que les frontières de sécurité de la Russie sont celles de l'ancienne Union soviétique. C'est, de manière plus inquiétante encore, le Professeur Loukine, président libéral de la Commission des Affaires étrangères à la Douma et ancien ambassadeur à Washington, déclarant que « la Russie est un pays qui n'a pas de frontières ». C'est le leader du premier parti russe, le Parti communiste, faisant campagne pour l'annulation des accords de 1991 qui mettaient fin à l'Union soviétique, ou ce sont les fuites organisées venant de l'état-major soviétique et menaçant les pays baltes d'invasion en cas d'élargissement de l'OTAN. Ne tombons pas, cependant, dans l'exagération selon laquelle rien n'aurait changé et l'Europe serait toujours confrontée à un empire russe menaçant, missionnaire et expansioniste. Autant l'optimisme sur la disponibilité des Russes à oublier la puissance impériale d'antan pour devenir une nation comme les autres et construire un État à l'occidentale semble pour le moins prématuré, autant les moyens de l'empire semblent absents, à commencer par le 
contrôle de Moscou sur son armée et ses provinces. La réalité première est tout de même celle de la décomposition et les ambitions autoritaires sont modérées moins par la conversion ou la résignation que par l'anarchie et l'incompétence. Quelles que soient les permanences ou les évolutions de l'âme russe, l'empire russe, presqu'autant que l'empire ottoman ou autrichien, est condamné à la fois par la montée des nationalismes et par celle du capitalisme occidental ou transnational. D'où un renversement par rapport à l'expérience historique : traditionnellement, c'est l'Europe de l'Ouest qui était fragmentée en États nationaux alors que l'Est, plus unifié, continuait, de Byzance à Moscou, la tradition impériale. On a pu dire que l'empire romain avait finalement rendu l'âme en 1991, l'empire d'Orient suivant enfin dans la tombe l'empire d'Occident, que certains voient d'ailleurs renaître sous les espèces pourtant peu grandioses de l'Union européenne. Ce qui est sûr, c'est que nous sommes plutôt dans une phase de déterritorialisation à l'Ouest, et de territorialisation à l'Est. On pourrait interpréter ce contraste comme un simple décalage historique : la phase de formation post-impériale des États nationaux, dépassée depuis longtemps à l'Ouest (et plus récemment au centre) y ferait place désormais au post-national et à l'intégration, alors qu'elle serait encore en plein essor à l'Est. Mais la part de vérité de cette vision - suggérée dans une certaine mesure par des auteurs aussi divers qu'E. Gellner et K. Pomian - ne doit faire oublier ni l'influence réciproque de l'Est et de l'Ouest, ni les facteurs transnationaux communs qui viennent troubler, voire bouleverser, toute succession ordonnée ou confortable des phases historiques. Il y a de puissants facteurs de re-territorialisation à l'Ouest et de déterritorialisation à l'Est.

6 Fragmentation et interdépendance La forte et éclairante image gellnerienne des « fuseaux horaires » ${ }^{11}$ risque de suggérer une concordance du temps et de l'espace là où ce qu'il s'agit de comprendre c'est précisément la complexité de leurs discordances et de leurs re-combinaisons. Un territoire national est caractérisé par une certaine combinaison de temps et d'espace. Pour une société traditionnelle, sédentaire et prénationale, l'horizon spatial est limité alors que l'horizon temporel, défini par l'histoire et par le mythe, remonte à un passé lointain ou vécu comme éternel. Le changement technique et la mondialisation tendent à élargir l'horizon spatial et à rétrécir de manière tout aussi spectaculaire l'horizon temporel. Nous sommes brutalement arrachés aux générations qui nous ont précédés et mis en contact immédiat avec des contemporains qui sont le produit d'histoires fondamentalement différentes de la nôtre. Le rapprochement dû aux communications et aux medias n'efface pas d'un coup de baguette magique la différence des niveaux de développement économique et politique, ni celle des croyances et des cultures. Au contraire, comme on l'a souvent montré, il nous amène tous à vivre simultanément des temporalités, partiellement communes et partiellement distinctes. Ce qui nous intéresse au point de vue des rapports entre Europe et territoire, ce sont les conséquences politiques de la déstabilisation qui en résulte à l'intérieur du continent, de ses sociétés, et de ses individus. A un premier niveau, le plus visible, on soulignera l'opposition entre sociétés urbanisées à l'Ouest, vivant d'abord dans l'espace et sociétés rurales ou en voie d'urbanisation à l'Est, vivant d'abord dans le temps. A un deuxième niveau, chacune est affectée par l'autre. Avant 1991, la balance des paiements yougoslave était dominée par le flux des touristes occidentaux et par celui des Yougoslaves travaillant à l'Ouest. Plus généralement, aujourd'hui encore, la présence des immigrants, et réfugiés dans les sociétés plus développées, celle des informations et des modèles de comportement de celles-ci transmis par les medias dans les sociétés moins développées rend leurs 
contacts et leur contamination réciproque inévitable. Mais le niveau le plus intéressant est le troisième, celui des réactions à cette situation, visant à rétablir la cohérence perdue. Là encore, la situation en ex-Yougoslavie constitue un exemple extrême qu'on ne peut généraliser mais qui éclaire la problématique générale. Premièrement, l'entreprise des médias gouvernementaux et avant tout celle de la télévision de Belgrade, consiste à effacer le passage du temps, à télescoper, au besoin par des montages visuels, les horreurs de 1941 et celles d'aujourd'hui, à projeter une image éternelle du Croate oustachi, de l'Allemand nazi et du Serbe héroïque et sacrifié. Deuxièmement, on essaie de remédier à l'incohérence par la séparation, d'où la fragmentation territoriale ou le nettoyage ethnique. Troisièmement, celui-ci entraîne, sous sa forme la plus extrême, un effort pour détruire la mémoire de l'autre, celle des cultures rivales ou de la ville cosmopolite : d'où la guerre aux mouvements religieux et culturels ou l'urbicide ritualisé dont parle Bogdan Bogdanovic ${ }^{12}$. La nostalgie du territoire au sens fort entraîne à la fois une guerre pour l'espace et une guerre pour la mémoire. Dans cette guerre, les mêmes changements technologiques qui promeuvent la mondialisation peuvent renforcer les solidarités ou les nostalgies territoriales. L'Internet et la baisse du coût des voyages facilitent le maintien des contacts entre diasporas et mère-patrie, et le rôle de ces diasporas va en général dans le sens de la radicalisation des conflits territoriaux. Mais, pour combiner deux formulations successivement à la mode, la guerre des cultures n'est pas la fin de l'histoire. Certes, plus le rythme du changement s'accélère, plus il produit la nostalgie de la stabilité et la recherche des racines; plus les nations sont ébranlées par la mondialisation, plus elles s'efforcent de reconstituer souveraineté, cohérence et clôture. Mais, justement, ces racines sont de plus en plus artificielles, les barrières partiellement infranchissables pour les hommes sont impuissantes à arrêter les biens et, encore moins, les messages. La simplicité, la stabilité et la fermeture sont aussi inaccessibles que sont insupportables la complexité, le changement et l'ouverture. Les compromis entre aspirations et contraintes contradictoires varieront, dès lors, selon les cas, c'est-à-dire à la fois selon les lieux et selon les dimensions des relations politiques, économiques, sociales et culturelles. Ainsi, dans le monde de l'après-guerre froide, on assiste à une globalisation accrue des relations économiques, alors que, du point de vue militaire ou stratégique, la disparition de la bipolarité entraîne plutôt une fragmentation du système international et une importance croissante des équilibres régionaux. Mais une fois de plus, la réalité est plus contradictoire que ne sembleraient l'indiquer ces tendances prédominantes. L'interdépendance économique produit des inégalités, des fractures, des cooptations et des marginalisations qui, à leur tour, peuvent entrainer des recompositions territoriales : c'est ce qui s'est produit en ex-Yougoslavie, pour la Slovénie et la Croatie; c'est une possibilité qui, de temps en temps, semble très présente en Espagne, en Belgique ou même en Italie. Pour les conflits armés, l'évolution va, bien sûr, des risques de guerre Est-Ouest à la réalité des guerres nationales et, plus encore, civiles. Le cas de l'Afghanistan en témoigne éloquemment. Mais, une fois de plus, l'Europe est plus compliquée. Pour revenir au cas de l'ex-Yougoslavie certes, personne ne pense, comme on l'aurait fait pendant la guerre froide, qu'il puisse produire une troisième guerre mondiale opposant Russes et Américains. Il reste que, fort heureusement d'ailleurs, ce sont des soldats américains et, plus généralement, extra-régionaux qui sont présents en Macédoine ou en Bosnie plutôt que des soldats grecs ou bulgares. Plus généralement, si la tendance post-bipolaire est au retrait américain et à la régionalisation de la sécurité européenne, ce sont les États-Unis qui 
exercent l'influence la plus décisive dans les Balkans et au Moyen-Orient, alors que l'Europe occidentale est présente avant tout sur le plan économique et humanitaire. De nouvelles configurations territoriales fluides et contradictoires, mais territoriales quand même à leur manière, sont ainsi en train de s'esquisser. Les États successeurs des empires cherchent à passer d'une intégration hégémonique qu'ils refusent à une intégration à l'Europe occidentale qu'ils souhaitent à travers une phase de territorialisation nationale. Mais cette belle dialectique a peu de chances de se dérouler de manière harmonieuse. Elle peut réussir sans trop d'accrocs dans certains cas, comme celui de la Slovénie qui est pratiquement sortie de l'espace yougoslave et en voie d'accès à celui de l'Europe centrale, et peut-être comme celui des républiques Baltes, en voie d'intégration à l'espace nordique qui peut constituer une étape vers l'Union européenne. Mais, sur le plan économique, les espaces ex-yougoslave et ex-soviétique sont le cadre premier de l'interdépendance pour les républiques dont l'aspiration à l'indépendance ou la recherche de dépendances de rechange se heurte aux réalités de la dépendance énergétique (comme dans le cas de l'Ukraine) ou aux courants industriels et commerciaux créés par des décennies de cohabitation. Surtout, l'interpénétration sociale, à la fois héritage du passé et accélérée par les nouvelles migrations, se heurte à un rejet qui s'exprime en tensions allant de la crise urbaine aux conflits religieux ou nationaux et au nettoyage ethnique. On tombe ainsi dans ce qu'on pourrait appeler les pathologies du territoire, déchiré entre forces centrifuges et centripètes, entre une diversité et une homogénéité, une logique de l'union, et une logique de la séparation, également impossibles à pousser jusqu'au bout. D'où la dialectique des minorités, des frontières et des migrations. Mais les compromis souhaitables, qu'expriment, notamment, les formules liées à l'État territorial fondé sur une conception civique, non religieuse et non ethnique, de la nation, ne sont pas plus faciles à mettre en oeuvre. Pour coexister pacifiquement sur le même territoire, il faut une autorité commune, ou une idéologie intégratrice, ou au contraire un esprit individualiste (inspiré au mieux par la tolérance et le respect mutuels, au minimum par l'indifférence et la dévalorisation d'enjeux comme la terre ou les ressources naturelles - ce que Gellner appelle le "fédéralisme de l'abondance ", fondé sur une société de consommateurs relativistes), ou les trois. Quand ils sont absents, le déplacement soit des frontières soit des populations peut devenir inévitable. Tout dépend alors, à la fois des modalités de ce déplacement et d'un pari sur l'avenir. Un théoricien de l'intégration comme Karl Deutsch a cependant souligné, dans un article peu connu, que la séparation, au moins temporaire, pouvait, dans certains conflits, constituer un moindre mal ${ }^{13}$. Mais quand elle se fait de manière unilatérale et violente, voire barbare, quand les nouvelles barrières politiques ne sont pas relativisées par un minimum de réciprocité et d'interpénétration transnationale au niveau des sociétés, elles conduisent à la dégradation intérieure des communautés séparées et portent le germe des vengeances et des conflits futurs. On pourra, ainsi, discuter de l'échange massif de populations entre la Grèce et la Turquie en 1923 ou de la partition de fait de Chypre pour soutenir soit qu'ils ont évité de nouvelles guerres gréco-turques, soit qu'ils ont entretenu la rancune entre les deux pays et fait, de certains territoires, comme la partie Nord de Chypre, sur des ghettos pathologiques.

7 Communauté et société : à la recherche d'une identité et d'une citoyenneté nonterritoriales Au livre III de la République, Socrate expose, après maintes précautions, ce qu'il appelle le "noble mensonge", à savoir le mythe d'autochtonie: il faudrait persuader « d'abord les dirigeants eux-mêmes et les militaires, ensuite le reste de la 
cité, qu'ils avaient été modelés sous la terre et qu'une fois que leur fabrication avait été terminée, la terre, qui est leur mère, les avait mis au monde ; et qu'à présent ils doivent délibérer au sujet du pays où ils sont, et le défendre contre quiconque l'attaque, comme si c'était là leur mère et leur nourrice, et penser aux citoyens comme à des frères nés comme eux de la terre ${ }^{14}{ }^{15}$. En d'autres termes, le mythe de la terre-mère ou de la mère-patrie est nécessaire pour fonder l'identité, c'est-à-dire à la fois l'unité et la distinction de la cité. Si la nation est un compromis entre une pseudo-famille (conception ethnique) et un pseudo-contrat (conception civique) le territoire est, lui aussi, un compromis entre un aspect mythique ou affectif et un aspect rationnel ou pragmatique : à la fois terre, ou héritage sacré, espace fonctionnel, et lieu de pouvoir, légitimé par les deux dimensions précédentes. Pour formuler la même idée de manière différente, il comporte la dimension de l'identité (pour laquelle la contiguiité apparaît comme le substitut de la consanguinité, fondant le droit du sol comme substitut au droit du sang), celle de l'autorité (c'est-à-dire du pouvoir légitime ou de l'État, comme instrument de contrôle, politique, juridique, policier et militaire sur une population définie par sa résidence), et celle de l'efficacité administrative, bureaucratique ou économique dans la gestion des mécanismes sociaux, à commencer par l'interdépendance. La force et la vertu de l'État territorial-national tenaient à la combinaison de ces trois dimensions. Aujourd'hui, tout ce que nous avons vu semble indiquer qu'elles tendent à se séparer. C'est ce que John Ruggie, dans l'article cité, appelle "the unbundling of territoriality" ${ }^{16}$. Des oppositions classiques entre l'intérieur et l'extérieur, le public et le privé, le politique et le social, le national et l'international sont mises en cause. Des formes nouvelles et ambiguës de communauté et de citoyenneté sub et transnationale s'esquissent, sans que pour autant la nation, l'État, le territoire, même amputés de certaines dimensions essentielles, soient prêts à leur laisser la place. Des entités abstraites et paradoxales (pour joindre les deux bouts de nos références concrètes: «l'objet non identifié » delorien qu'est l'Union Européenne, la Bosnie-Herzégovine des accords de Dayton, État souverain composé de deux « entités » qui peuvent avoir des liens spéciaux avec des États voisins différents), des populations aux statuts ambigus ou incomplets (réfugiés, tsiganes, résidents permanents, citoyens européens), des lieux eux aussi à statuts spéciaux ou obscurs, depuis le camp de concentration jusqu'à la "zone de sécurité » en passant par les centres de tri, les " zones grises ", les enclaves ou les zones à statut spécial placées sous administration internationale) prolifèrent, sans parler des différents réseaux à tous les sens du terme. L'important est que rien de tout cela ne constitue un ordre international ou, moins encore, européen. On peut imaginer un monde dominé par des réseaux, où des équivalents fonctionnels de l'État et du territoire se substituent à ces derniers du point de vue économique et militaire, voire (encore que plus difficilement) administratif ou policier. On ne voit guère comment ils pourraient produire un équilibre entre communauté et société, entre identification affective et efficacité fonctionnelle où les besoins contradictoires de l'âme et de la société humaines trouveraient à se satisfaire. Si toutes les identités et les communautés, les racines et les frontières concrètes sont construites et passagères, le besoin d'identité et de communauté, celui de distinguer entre "nous » et «les autres » ne l'est pas. Nié ou bafoué, il se venge de manière destructrice. Bertrand Badie a bien raison de lier la résurgence de la terre, de l'ethnicité, de l'identification par le sang, au déclin du territoire. Il est moins convaincant (et peut-être moins convaincu) quand il esquisse des recompositions et quand il fait appel à un respect quelque peu désincarné de l'autre qui 
ne se fonderait ni sur le sang, ni sur le sol, ni sur la croyance ${ }^{17}$. Peut-être l'éclatement ou la criminalisation des communautés concrètes ne nous laissent-ils pas d'autre recours qu'une morale de type kantien. Encore faut-il se rappeler les critiques hégéliennes sur le formalisme du contrat ou, plus près de nous, les flèches de Péguy contre le kantisme qui a les mains pures mais qui n'a pas de mains. Entre une rationalité formelle et des identités substantielles écartelées ou cherchant à retrouver une unité dans le délire, le moins qu'on puisse dire est que les médiations font cruellement défaut. En ce sens, les deux termes de notre titre, Europe et territoire, indiqueraient deux cas particuliers d'une réalité qui est celle de notre époque, qu'Auguste Comte aurait appelée «critique » par opposition à « organique »: le temps des dissociations sans recomposition, ou des contradictions sans synthèse.

\section{NOTES}

1. M. F. Durand, « Entre territoires et réseaux », in B. Badie, C. Wihtol, de Wenden (dir.), Le défi migratoire, Presses de la FNSP, 1994, p. 155.

2. P. Manent, « Note sur l'individualisme moderne », Commentaire, XVIII, 70, été 1995, p. 263.

3. V. Andreff, dans le même ouvrage.

4. John G. Ruggie, « Territoriality and beyond : problematizing modernity in international relations », International Organization, 47, 1, hiver 1993, p. 172.

5. Ibidem.

6. B. Barret-Kriegel, Etat de droit et empire, république et démocratie.

7. R. Koselleck, « Diesseits des Nationalstaats, Föderale Strukturen der Deutschen Geschichte », Transit, 7, printemps 1994.

8. T. Garton-Ash, In Europe's Name, Londres, J. Cape, 1993, pp. 393-410.

9. Cf. R. Aron, « Les racines de l'impérialisme allemand », in Chroniques de Guerre, Gallimard, 1990, p. 606.

10. L. Delattre, «L'Allemagne entre normalité et puissance », Le Monde, 31 déc. 1995, 1er janvier 1996.

11. E. Gellner, « Nationalisms and the New World Order », in C. Kaysen and L. Reed (eds)., Emerging Norms of Justified Intervention, Cambridge, 1993, pp. 152-153.

12. B. Bogdanovic , "L'urbicide ritualisé », in V. Nahoum-Grappe, Vukovar-Sarajevo, La guerre en ex-Yougoslavie, Paris, ed. Esprit, 1993, pp. 33-38.

13. K. Deutsch, «Space and Freedom », International Political Science Review, 1985.

14. Platon, La République, traduction de Pierre Pachet, Folio, Gallimard, 1993, p. 194.

15. Ruggie, loc. cit.

16. Ruggie, loc. cit.

17. Cf. B. Badie, La fin des territoires. Essai sur le désordre international et sur l'utilité sociale du respect, Paris, Fayard, 1995. 
INDEX

Index géographique : Europe

Mots-clés : Théorie politique, territoire(s) et territorialité, identité, crises politiques 